\title{
A Turn Towards the Digital. An Overview of Swedish Heritage Information Management Today
}

Daniel Löwenborg, Maria Jonsson, Åsa Larsson and Johan Nordinge

\section{Summary}

Heritage management in Sweden has undergone a substantial transformation in recent decades. The process of monitoring and managing heritage information has become increasingly digital, relying on interconnected systems to monitor registered archaeological remains to manage investigations and contract archaeology excavations. This also has to work together with the digital systems of the County Administrative Boards that administer all permissions for excavations. Current developments deal with archiving and dissemination of reports, and documentation from fieldwork.

Documentation of archaeological excavations has predominantly been digital for the past 20 years, which brings both possibilities and challenges in making sure the information will adhere to the FAIR Principles. This article outlines some of these developments and exemplifies the possibilities of reusing legacy data through the Urdar project.

\section{Contract Archaeology in Sweden}

Archaeology in Sweden underwent substantial changes in the 1990s. Before this, almost all excavations were undertaken by either the archaeological unit of the National Heritage Board (Riksantikvarieämbetet UV), which had offices in several cities, or by regional and local museums. Universities were mostly only involved in research excavations. The National Heritage Board (NHB) was also responsible for national surveys between 1937 and 2000, aimed at identifying ancient sites. The survey work was mainly aimed at generating information for landowners, since historic and prehistoric sites are not allowed to be damaged, even when they are on private land.

This strong legal protection resulted in logistical problems for society, as the rate of infrastructure development increased from the 1960 s onwards. Combined with political 
changes in the 1980s that favoured deregulation, the Historic Environment Act was adjusted in the late 1980 s to allow for private competitors. The establishment of archaeological companies outside of museums took off in the 2000s and at the same time regional and local museums found it increasingly difficult to compete for excavation projects and several have completely disbanded their archaeological units - some of which then started companies of their own (Larsson and Löwenborg 2020).

A decision whether an ancient site is to be excavated and by whom is solely decided by the local County Administrative Board (CAB). The cost must be covered by the developer in question, but the developer has no say in the requirements or the evaluation of the tenders - only CAB does. The role of the National Heritage Board developed alongside this. The NHB writes general rules and requirements (Föreskrift) for contract archaeology, which CAB has to adhere to when offering a tender. The NHB has oversight to make sure $\mathrm{CAB}$ follows these regulations and can appeal cases to the Courts.

While the archaeological unit at the NHB operated as an independent company, kept apart from the agency that dealt with regulations and oversight, the situation was less than ideal. In 2015 the company was transferred to the National Historical Museums, to become an independent part of that organisation instead. It is still the biggest employer of contract archaeologists in Sweden, with offices in several cities, but it is now competing with 50-70 small to medium-sized archaeological companies (including those operated by museums). There are also a few fairly large private companies, though these tend to be concentrated within particular regions: Central, West and South Sweden respectively. The system is illustrated in Figure 1.

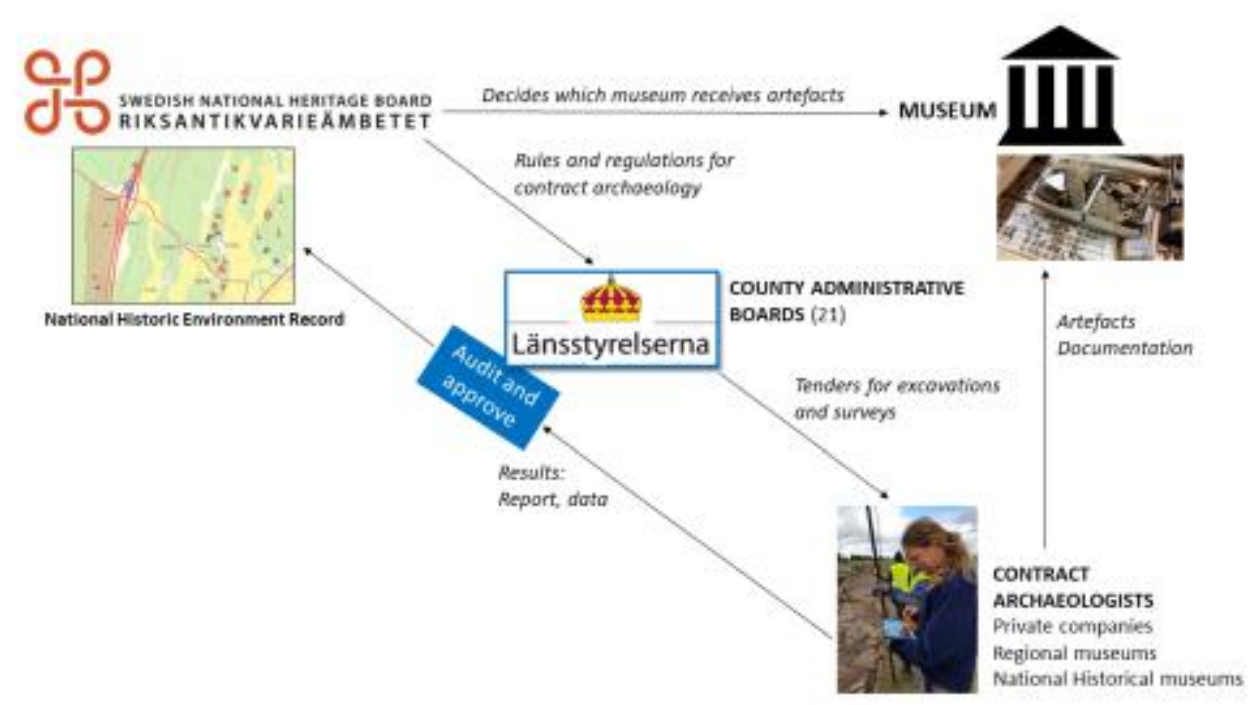

Figure 1: The Swedish system for contract archaeology illustrated and simplified. The National Heritage Board sets rules and regulations for the system. The County Administrative Board ( $\mathrm{CAB}$ ) can approve the excavation of a site, whether on private or public land. Developers have to cover the cost of contract archaeologists decided by $\mathrm{CAB}$. The results must be approved by $\mathrm{CAB}$ before they become public in the Historic Environment Record. Artefacts and analogue documentation are delivered to a museum. Illustration: Åsa Larsson, CC-BY 
Despite the loss of the archaeological unit, the NHB still has a very central role to play with regard to contract archaeology, apart from overseeing regulations. The NHB is assigned the task of keeping a national Historic Environment Record (HER) with information about ancient and historic sites to help CAB in their decision making. This includes information about sites that have already been excavated and removed, as they are vital to understanding the landscape. For instance, CAB often orders surveys to be done by contract archaeologists in areas scheduled for development, in order to identify previously unknown sites, including studying archival information. For this reason, the NHB Archive receives a copy of every report produced by contract archaeology (see below).

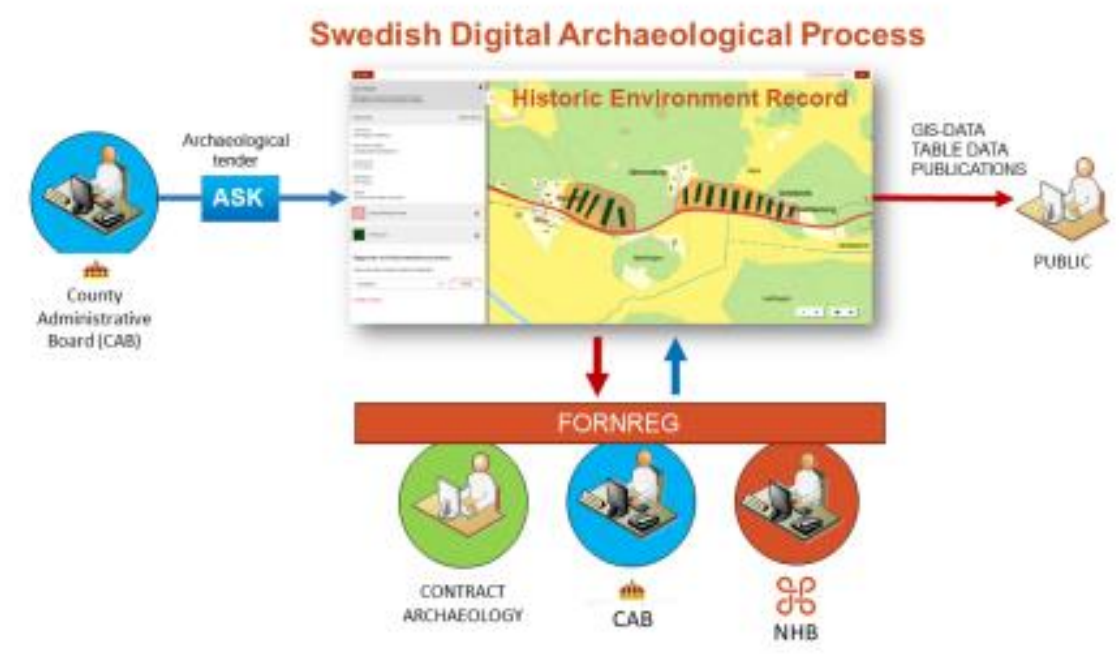

Figure 2: The Digital Archaeological Process implemented in Sweden in 2018. The County Administrative Board system (ASK) sends administrative data about planned archaeological excavations and surveys to the Historic Environment Record (HER). Via the web tool Fornreg the archaeologists can update HER with new information and upload the finished reports. Administrators at CAB can make the new data public and $\mathrm{NHB}$ can add information about which museum receives the artefacts. All content is open for public use through web search Fornsök and the Open Data Portal. Illustration: Åsa Larsson, CC-BY

The analogue predecessor (Fornminnesregistret) of the Historic Environment Record was digitised and made public on the internet in 2006-2008. Starting in 2019 the entire process of updating information in the HER is fully digitised through the web tool Fornreg (Larsson et al. 2017; Larsson and Löwenborg 2020; see also Figure 2). Contract archaeologists can now update the data in the Ancient Sites and Monuments Record directly. CAB has to review and approve these changes before they are made public. For more on the current process, see below. 


\section{Archiving Archaeological Documentation}

\subsection{Physical documentation}

Information about archaeological excavations have been sent to the NHB Archive almost from the start of the discipline, though it would take until the professionalisation in the 1950-60s for proper reports to be produced. Copies of these reports are required to be sent to the NHB, though in 2019 the rules changed so that only digital copies are now accepted (see below).

Original documentation from the excavation has to be delivered to a museum together with the artefacts. For most of the 20th century, this was just common sense, as museums were also the ones doing most of the excavation. The National Heritage Board and the Swedish History Museum were part of the same organisation until the late 20th century, when the National Historical Museums became a separate agency. This meant that almost all the documentation from excavations done by the NHB archaeologists were kept at the NHB Archive (ATA - Antikvarisk Topografisk Arkiv) since it was also the archaeological archive for the History Museum, even after the partition. However, when the archaeological unit was finally transferred to the National Historical Museums in 2015, making the partition more substantial, the archaeologists no longer sent their physical documentation to the NHB. Of course, this is also because almost all of the documentation is now generated digitally, and is no longer physical, which has created its own issues (see below).

The rapid growth of private contract archaeology companies in the early 21st century created a challenge for the system, as these, unlike museums, are not required by law to keep an archive. The regulations regarding the delivery of documentation are a bit unclear and mostly recommendations rather than strict rules. Besides, the actual delivery of documentation is not something $\mathrm{CAB}$ follows up on, as it is only done once the NHB decides which museum receives the artefacts - which only happens once the report is published. The decision process by the NHB can take several years, which also increases the risk of documentation being forgotten in the meantime. The newly digitised process will significantly decrease the time between the finished report and accession of artefacts, but whether physical documentation is archived properly remains to be seen.

\subsection{Digital documentation}

The archaeological unit at the Swedish National Heritage Board developed an 'Intra-site information system' for digital documentation, which was released as the commercial software Intrasis in 2001 (Anund and Lagerlöf 2009, 90). Ownership of Intrasis was transferred to the National Historical Museums in 2015 when the contract archaeology unit was separated from the NHB. There is no requirement to use Intrasis in Swedish contract archaeology; though many companies do so, some have developed their own software or use a combination of other products.

Unfortunately, the system for archiving said documentation has not kept up with technological changes. The National Heritage Board did not produce formal regulations 
(Föreskrift) regarding archiving of archaeological documentation in general until 2007 (KRFS 2007, 2), and initially it made no specific mention of digital documentation, only digital reports. It was also only a recommendation at first, not a regulation. This was partly due to the fact that as long as the archaeology unit belonged to a public organisation such as a museum, there were already routines in place to deal with archiving. The regulation was updated in 2015 to better reflect the current reality and KRFS 2015:1 \$16 stipulates that a public archive has to be chosen by CAB as part of the contract archaeology process. $\$ 36$ also specifies that both analogue and digital documentation should be archived.

However, there are no detailed requirements as to what documentation has to be delivered in every case; it is very much up to the contract archaeologists to make the selection. Although some archaeologists have been sending digital documentation to the NHB Archive even before the new regulations came along, often as CDs with files containing appendices and photos for the reports, there have been no routines in place for preserving these in sustainable formats or making them easily accessible to the public. The Swedish History Museum asks for Intrasis-databases to be delivered with the finds they receive and stores these on their servers. For companies using other software for documentation, a spreadsheet of the finds is delivered, but not necessarily any other digital documentation.

Few, if any, other museums have a system in place to archive digital documentation (GIS-files, databases, digital photos, etc.). Since the publicly funded part of the museum should not overlap with the contract-funded part, the documentation is usually kept on the latter's servers. If the museum disbands its archaeological unit, as is becoming increasingly common, knowledge of what data is kept where may be lost, and it may even be deleted. As private archaeological companies have no requirement to have an archive, their data are completely lost if and when they close down. In recent years, the Swedish National Data Service (SND) has been including digital archaeological information from excavations in the data hosted by SND. While SND focuses on research data from universities, they have also included some contract archaeology data that was collected in collaboration with Uppsala University (see Jakobsson, this issue).

\section{There is a Will but not a Way - yet}

As the digital transformation picked up speed within contract archaeology in the 2010s, the system fell even further behind reality. Contract archaeologists now produce massive amounts of data and digital documentation each year but have nowhere to store it long term or make it publicly accessible. The only exception is that artefact lists from 2019 and onwards are to be uploaded as CSV-files to the Historic Environment Record maintained by the NHB, together with the reports as PDF files. The artefact lists are prioritised because they are needed to facilitate the decision-making process within the $\mathrm{NHB}$ as to whether the national or a local museum should receive them, and also facilitate the process of CMS registration for the museums.

The question of preservation of digital documentation is very much an active issue at the Swedish National Heritage Board. The developments made to the digital Historic Environment Record (HER) in 2014-2019 as part of the Digital Archaeological Program (Larsson et al. 2017) means there is now a cohesive infrastructure that could act as a repository for archaeological documentation. The HER now contains a record of 
archaeological excavations and surveys and, more importantly, it is also integrated with an OAIS e-archive capable of long-term preservation of digital content.

The new digital process means that any archaeological project decided by the County Administrative Boards is automatically added to HER since their digital system is linked to the HER. The web tool Fornreg allows contract archaeologists to update information about old and new sites, add geometries of excavated trenches, and upload reports and artefact lists, which are then stored in the repository. Everything is publically available through the website Fornsök and the search interface for the e-archive Forndok (Figure 3).

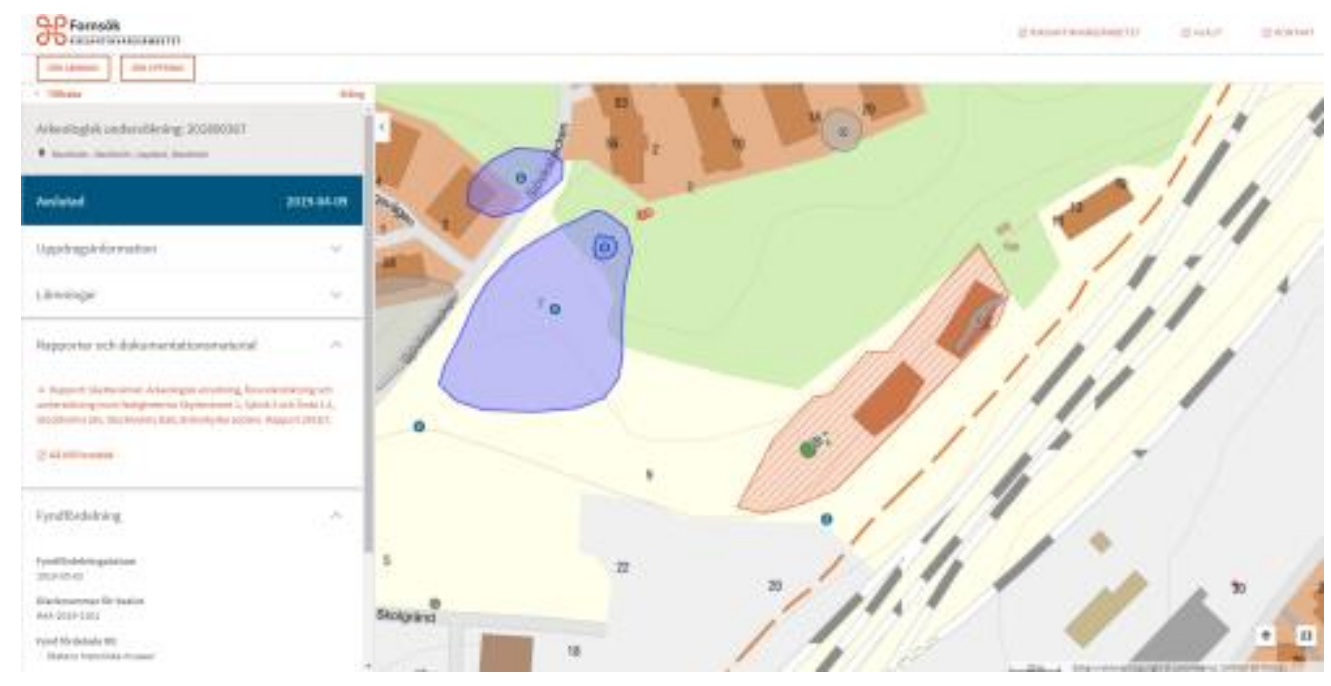

Figure 3: The public web interface for the Historic Environment Record: Fornsök. Red geometries marked ' $R$ ' are protected sites, blue are historical sites with less degree of protection, grey are sites that have been destroyed or excavated. The striped red geometry is the area of an archaeological project. On the left is the info card, with a link to the report (PDF) and information about which museum received the artefacts

Compliance with guidelines among archaeologists is generally high, since there is a common digital tool and system for administering the projects. The County Administrative Board will only change the status of a project to 'concluded' once all the required information is registered. Since $C A B$ is responsible for all tenders and assignments involving excavation, and for the majority involving surveys, most contract archaeologists will take very good care to follow the requirements in these or risk losing future work. The CAB tenders in turn follow the national regulations and recommendations laid out by the NHB. However, the lack of clear guidelines regarding digital documentation, and especially the lack of repositories capable of archiving such content properly, make the situation bleaker than it may appear at first sight. It is not lack of compliance that is at issue, it is lack of rules, guidelines and infrastructure.

There is an urgent need to develop the repository at the NHB so that the County Administrative Boards can update their guidelines to archaeologists of where to deposit their digital documentation and how. In order to do so, we need a better understanding of how to save born-digital documentation in ways that are both preservable and reusable. This is one reason that Project Urdar - $A$ research infrastructure for archaeological excavation data was recently initiated as a cooperative effort between Uppsala University and the NHB (see below). 


\section{The Swedish National Heritage Archive and the FAIR Principles}

\subsection{Findable}

The digital objects in the NHB Archive are to a large extent findable - they are assigned unique and persistent identifiers, and they are searchable in the archive's public web interface Forndok. Reports and finds lists can also be found through Fornsök, the mapbased web interface of the Historic Environment Record (HER), by searching for the excavations either by location or administrative criteria.

Parts of the archival information are findable through $\mathrm{SOCH}$, which is the national aggregator for cultural heritage data developed and maintained by the NHB. Metadata for documents and images are harvested, but not yet structured data such as finds lists.

Digital objects and datasets stored in the e-archive are assigned identifiers according to NHB's syntax for persistent identifiers. The metadata elements themselves are for the most part not described by identifiers. For example, author names are stored as text values, but not with identifiers from a resource that describes authors. Identifiers for metadata elements are used to a larger extent when it comes to geographical metadata and metadata elements holding relationships to other digital objects within HER.

Both in HER and the e-archive, the metadata describing an object includes an identifier pointing to that object. For example, a digital object in the archive includes a URI for the digital object itself, as well as the metadata describing it. A digital object that is stored in the archive consists of both metadata and file/s.

Digital objects stored in the NHB archive are indexed in the archival system and searchable through the archival system's public web interface, which is publicly accessible to everyone. Not all metadata is exposed publicly, and is therefore not searchable. Metadata from the archive are also harvested by SOCH for archival objects of the resource types Image and Document. SOCH has an open web-API and a public web interface via Kringla.nu.

\subsection{Accessible}

Metadata can be retrieved from the archive via OAI-PMH, which is the protocol used by $\mathrm{SOCH}$ to harvest metadata. The archives' OAI-PMH service used by $\mathrm{SOCH}$ is not publicly accessible. The metadata can also be retrieved from the archive by their identifier via HTTPS, where metadata is publicly accessible as JSON via the 'Document API'. Data files can also be retrieved this way, through a URL based on the digital object's identifier. Not all data are provided this way - sensitive data or copyrightprotected data are only available on request. Requests can be made via an order function, which is accessible in the public web interface for Forndok. Another way to request this data is by telephone or by emailing the $\mathrm{NHB}$.

The Open Data Portal of the NHB allows the public to access and download the entire ancient sites and monuments record, as well as the record of excavations and surveys, 
as geopackages or shapefiles. They can also be accessed as WMS. This data export of metadata, text information and GIS-data does not include the content stored in the earchive repository.

\subsection{Interoperable}

Interoperability is low. Even though metadata are shared through open protocols, and represented as JSON in the NHB's document API, metadata are not expressed in formal, shared or well-documented metadata standards such as Dublin Core or CIDOCCRM.

When data are described, controlled vocabularies do not use persistent identifiers for the most part, which of course limits the possibilities of interoperability when the metadata is harvested from the archive by $\mathrm{SOCH}$. Identifiers are used for some vocabularies, e.g. licensing and rights statements.

Qualified references cannot be achieved within the archival system at the NHB. When metadata is shared in $\mathrm{SOCH}$, however, qualified references are made possible, which makes it possible to describe, for instance, that an image visualises a certain ancient site or monument, or that an identifier is of a certain type.

\subsection{Reusable}

The metadata is rich primarily in the sense that they allow for discovery through the use of contextual identifiers as well as descriptive metadata. However, the FAIR Principles for reusability state that rich metadata should also describe the context under which the data were generated. For instance, for what purposes the data were generated or collected, the limitations of the data, the version of software used to collect the data, or how the data has been processed. This is not included in the metadata published by the $\mathrm{NHB}$, at least not in a machine-readable way. The key to understanding the data's provenance is usually a reference to additional documentation, such as a report. The reference consists of an identifier, usually a registration number for the record or an identifier from HER. This reference is not linked or explained, which requires the user to have prior knowledge of what the references mean and how to use them. There is also a risk that the 'plurality' aspect is being overlooked, as datasets and metadata are often tailored to the needs of certain target groups (the creators of the data, rather than the users).

All digital objects are provided with a licence before archiving. Creative Commons licences are used for openly shared data. Metadata is released with the licence CC-0. 


\section{Current Development in Swedish Digital Archaeology, the Urdar Project as an Example}

As discussed above, the National Heritage Board (NHB) has been responsible for a substantial part of archaeological excavations carried out in Sweden between 19592014. The contract archaeology unit at NHB developed their own site documentation software, Intrasis, that came into use around the year 2000. Intrasis has also been licensed to other archaeology firms active in Sweden. This means that much of the current digital documentation about excavations comes in a structured format from the same software solution.

While the use of Intrasis made field documentation and post-excavation work more efficient, the born-digital data have not been reused for aggregated analysis outside the organisation where the data was created. The main reason for this is that there has not been any common digital repository hosting the data, as discussed above (though see Jakobsson, this issue). The documentation from excavations has thus not been reused in research, and the full potential of using the information from excavations to create new knowledge is limited. It has therefore been difficult to make use of digital technology for the type of research that such data would enable.

To start addressing the situation regarding the inaccessibility of data from excavations, the Urdar project was started in 2020. Urdar is a four-year research infrastructure project, undertaken in collaboration between Uppsala University and the NHB, with funding from Riksbankens Jubileumsfond. The aim is to preserve data in open and archivable formats, make it findable and freely accessible, and to explore some of the research potential - reusability. About 3700 databases from excavation projects are included in this pilot study, where each project represents anything from one or a few unproductive trenches to large excavations with tens of thousands of archaeological features, finds and samples.

While the data are created using the same software, there are still significant differences in how excavated features have been recorded and described, and there has been no standardised ontology used for all projects. The Intrasis format is proprietary so it needs to be converted to open formats before archival use with other software. As with all GIS data, there are also issues with coordinate systems and data quality that need to be addressed. Nonetheless, these data represent a large amount of high-quality information with the results from many excavations, and as such has considerable potential for reuse in both research and heritage management. In Norway, the ongoing ADED project (Archaeological Digital Excavation Documentation), has a similar scope since the Intrasis software package has also been used for archaeological excavations there.

The Urdar project will also do a small digitisation test, focusing on one or two parishes in Sweden. Analogue documentation available in the Topographic series of the NHB Archive, spanning the 19 th to the 21 st centuries, will be digitised. The purpose is to increase the accessibility of the documentation and also develop methods for largescale digitisation initiatives further on. The ambition is to ensure that analogue documentation and born-digital documentation can be combined, and together provide 
an overview of more of the total amount of archaeological excavations carried out in Sweden. This will benefit heritage management, and also enable a range of new research questions that can be answered by analysing the results of excavations on a landscape level, using modern technology for spatial analysis to learn more about the social processes behind the material.

\section{Conclusion}

There has been positive development in Sweden regarding the digital archiving of archaeological data for a long time. At the moment there are several ongoing projects on the way to support better integration of these functions and different systems. With a long tradition of working digitally in archaeology, Sweden has had a good starting point for developing these platforms. Another important aspect is that the combination of the $\mathrm{NHB}$ and the regional County Administrative Boards has allowed Sweden to centralise coordination of heritage management. This means that there are fundamental institutions in place to ensure consistency in management, and regulations that are of great value when systems are developed for digital heritage information.

All aspects of archaeological work in Sweden are now, at least to some extent, digital. This spans fieldwork and recording to writing and submitting reports, research and knowledge production, and now also archiving. With this transition towards a digital workflow, it is also possible to see a change in attitude among archaeologists, with increasing openness to the benefits of sharing information and better access to data benefiting everyone in the discipline. In the long term, this will probably affect how field archaeologists document excavations and think about how they describe their data so that others can reuse this information in other contexts. Ideally, this should also encourage archaeologists to use accepted standards for their documentation, and enhance the use of metadata included in the datasets, as the importance and benefits to good practice become apparent.

\section{Acknowledgements}

This work was supported by Riksbankens Jubileumsfond as part of Project Urdar, Grant number In19-0135:1.

Anund, J. and Lagerlöf, A. 2009 'Den vetenskapliga revolutionen. 1990-talet' in L. Ersgård (ed) UV 50 år, Riksantikvarieämbetet, Stockholm, 79-

136. http://urn.kb.se/resolve?urn=urn:nbn:se:raa:diva-5573

Larsson, $\AA$. and Löwenborg, D. 2020 'The digital future of the past - research potential with increasingly FAIR archaeological data' in C. Hillerdal and K. Ilves (eds) Reimagining Periphery. Archaeology and text in northern Europe from Iron Age to Viking and Early Medieval Periods, Oxford: Oxbow Books. 61-

70. https://doi.org/10.2307/j.ctv138wt08.8 
Larsson, A..M., Smith, M., Sohlenius, R. and Klafver, T. 2017 'Digitising the archaeological process at the Swedish National Heritage Board: producing, managing and sharing archaeological information', Internet

Archaeology 43. https://doi.org/10.11141/ia.43.6 